\title{
Abelhas (Hymenoptera: Apoidea) visitantes das flores de urucum em Vitória da Conquista, BA
}

\author{
Bees (Hymenoptera: Apoidea) visitors of the annatto flowers in Vitória da \\ Conquista, Bahia State, Brazil
}

Augusto Jorge Cavalcante CostaI Fábia Guimarães-Dias ${ }^{I I}$ Raquel Pérez-Maluf ${ }^{I I I}$

\section{-NOTA-}

\section{RESUMO}

O urucum é um arbusto da família Bixaceae, utilizado na fabricação de corantes naturais para a indústria alimentícia e cosmética. No Brasil, somente nos últimos 15 anos, houve maior interesse pelo cultivo, pois se tornou uma alternativa agrícola promissora. O presente trabalho teve por objetivo identificar as abelhas visitantes das flores do urucuzeiro em Vitória da Conquista, BA. O trabalho foi conduzido no campo experimental da UESB, em uma lavoura do tipo cultivado Peruana Paulista. A coleta das abelhas visitantes foi feita na época principal de floração do urucueiro: março/ abril, das 6h às 18h. Foram coletadas 3019 abelhas de 22 espécies, com predominância na visitação das $8 \mathrm{~h}$ às $14 \mathrm{~h}$ em relação ao número de indivíduos e número de espécies capturadas. As espécies mais freqüentes foram: Trigona spinipes (Fabricius), Apis mellifera L., Schwarziana quadripunctata (Lepeletier) e Tetragonisca angustula (Latreille). Espécies de maior porte, como Xylocopa frontalis (Olivier), Bombus morio (Swederus) e Eulaema nigrita Lepeletier, consideradas como eficientes na polinização da cultura do urucum, não foram abundantes neste estudo.

Palavras-chave: interação inseto-planta, diversidade.

\section{ABSTRACT}

Annatto is a shrub from Bixaceae family, which natural pigment (annatto) is widely used in food and cosmetic industries. In Brazil, the interest for this crop started in the last fifteen years, once it became a promising agricultural alternative. This study was aimed at identifing visitor bees of annatto flowers at the agriculture region of Vitória da Conquista (BA). The research was carried out in the experimental field of $U E S B$, in an experimental plot planted with the $c v$. Peruana Paulista. The visitor bees were collected during the main blooming period: March/April, between 6:00h and 18:00h. A total of 3,019 bees from 22 species was collected, with higher visitation during the period from 8:00 to 14:00h, regarding the number of individuals and species. The species most frequent were Trigona spinipes (Fabricius), Apis mellifera L., Schwarziana quadripunctata (Lepeletier) and Tetragonisca angustula (Latreille). Larger species as Xylocopa frontalis (Olivier), Bombus morio (Swederus) and Eulaema nigrita Lepeletier considered efficient pollinators of the urucum culture, were not abundant in the evaluated field.

Key words: insect-plant interaction, diversity.

O urucum ou urucu (Bixa orellana L.) é um arbusto perene da família Bixaceae, originário da parte norte da América do Sul e constitui a única espécie dessa família. Apresenta flores pentâmeras, com muitos estames e inflorescências paniculadas terminais de coloração rósea (JOLY, 1991). De acordo com GÓMEZ (1980), no urucum, não ocorre autopolinização, prevalecendo a xenogamia, com pouca ocorrência de geitonogamia, devido à protandria. VILARES et al. (1992) consideram que um dos fatores que influenciam a produtividade e qualidade dos grãos do urucueiro é a alta taxa de variabilidade genética de plantas do mesmo tipo cultivado, incrementada pelo elevado índice de polinização cruzada. Considerando o interesse pelo estudo das relações entre abelhas e pela polinização de plantas comercialmente exploradas, o objetivo deste trabalho foi fazer um estudo sobre as espécies de abelhas visitantes na cultura do urucum em Vitória da Conquista-BA, na época de sua maior floração, visando a contribuir para o melhor manejo na dinâmica reprodutiva desta cultura.

IPrograma de Pós-graduação em Agronomia, Universidade Estadual do Sudoeste da Bahia, Vitória da Conquista, BA, Brasil.

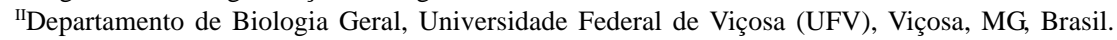

IIIDepartamento de Ciências Naturais, Universidade Estadual do Sudoeste da Bahia (UESB). Estrada do Bem Querer, Km 04, 45083-

900. Vitória da Conquista, BA, Brasil. E-mail: raquelperezmaluf@gmail.com. Autor para correspondência. 
O trabalho foi executado no campo experimental da Universidade Estadual do Sudoeste da Bahia, em Vitória da Conquista, Bahia. Foi utilizada uma lavoura de urucueiro com o tipo cultivado Peruana Paulista, com idade aproximada de oito anos. O período de amostragem compreendeu a florada principal do urucum, que, em 2004, estendeu-se entre os meses de março e abril. O horário da abertura floral varia em função da localidade, mas ocorre nas primeiras horas da manhã, em torno das 4 h às $5 \mathrm{~h}$, podendo estender-se até às 8h. Segundo ALMEIDA \& PINHEIRO (1992), o estigma fica receptivo desde o início da antese, juntamente com a maturação das anteras, e ambos permanecem assim cerca de seis a sete horas após a abertura da flor. Em função destas características, foram estabelecidas seis faixas de horários para coleta: $6 \mathrm{~h}$ $8 \mathrm{~h}, 8 \mathrm{~h}-10 \mathrm{~h}, 1 \mathrm{~h}-12 \mathrm{~h}, 12 \mathrm{~h}-14 \mathrm{~h}, 14 \mathrm{~h}-16 \mathrm{~h}$ e $16 \mathrm{~h}-18 \mathrm{~h}$. Coletaram-se com rede entomológica as abelhas presentes nas flores. Para o estudo da estrutura da comunidade de abelhas que visitam as flores do urucum, foram estimados a riqueza de espécies (S), o índice de diversidade de Shannon (H'), a eqüitatividade (J), a freqüência relativa e a constância (calculada a partir do total de coletas contendo a espécie i, sobre o total de coleta. Classificam-se as espécies em constantes - W: presentes em mais de $50 \%$ das coletas, em acessórias - Y: presentes entre 25 e 50\% das coletas e em acidentais $-\mathrm{Z}$ : presentes em menos de $25 \%$ das coletas) e em dominância (freqüência relativa superior a 1/S) (SILVEIRA NETO et al.,1976). Também foi estimada a sobreposição de nichos entre as espécies predominantes a partir da porcentagem de sobreposição P, estimada pela fórmula: $\mathrm{P}_{\mathrm{jk}}=\Sigma\left(\min \mathrm{p}_{\mathrm{ij}}, \mathrm{p}_{\mathrm{ik}}\right) 100$, onde $\mathrm{p}_{\mathrm{ij}}, \mathrm{p}_{\mathrm{ik}}$ corresponde à proporção do recurso i do total de recursos utilizados pelas espécies j e $\mathrm{k}$ (KREBS, 1989).

Foram coletadas 3.019 abelhas, com riqueza de 22 espécies, havendo predominância de 21 espécies nativas brasileiras, com 2.197 indivíduos, sobre Apis mellifera, com 822 indivíduos (Tabela 1). A diversidade estimada pelo índice de Shannon (H’) é de 1,58, com um índice de equitatividade (J) de 0,51. As espécies mais abundantes foram: Trigona spinipes, Apis mellifera, Schwarziana quadripunctata e Tetragonisca angustula, que também foram classificadas como constantes e dominantes (Tabela 1). Estas quatro espécies contribuíram com $94,6 \%$ do total de indivíduos coletados e apresentam uma importante porcentagem de sobreposição de atividade de forrageamento (P) (Tabela 2), sugerindo uma partição

Tabela 1 - Número total de abelhas coletadas em flores do urucum (Bixa orellana L.), em Vitória da Conquista, entre março e abril de 2004. (N: número total de indivíduos; F: freqüência relativa; C: constância, sendo constante (W), acessória (Y) e acidental (Z); S: riqueza; D: dominância, sendo dominante (d) e não-dominante (nd); H’: índice de diversidade de Shannon e J: Eqüitatividade).

\begin{tabular}{|c|c|c|c|c|c|}
\hline Família & Espécies & $\mathrm{N}$ & $\mathrm{F}$ & $\mathrm{C}$ & $\mathrm{D}$ \\
\hline Andrenidae & Oxaea flavescens Klug & 1 & 020003 & $\mathrm{Z}$ & nd \\
\hline \multirow[t]{16}{*}{ Apidae } & Apis mellifera L. & 822 & 27,23 & $\mathrm{~W}$ & $\mathrm{~d}$ \\
\hline & Bombus morio (Swederus) & 14 & 0,46 & $\mathrm{Y}$ & nd \\
\hline & Centris (Centris) aenea Lepeletier & 3 & 0,10 & $\mathrm{Z}$ & nd \\
\hline & C. (Melacentris) Moure cfr. collaris & 8 & 0,26 & $\mathrm{Y}$ & nd \\
\hline & C (M.) obsoleta Lepeletier & 6 & 0,20 & $\mathrm{Z}$ & nd \\
\hline & Cephalotrigona capitata (Smith) & 1 & 0,03 & $\mathrm{Z}$ & nd \\
\hline & Epicharis flava (Friese) & 1 & 0,03 & $\mathrm{Z}$ & nd \\
\hline & Eulaema nigrita Lepeletier & 2 & 0,07 & $\mathrm{Z}$ & nd \\
\hline & Exomalopsis analis Spinola & 23 & 0,76 & $\mathrm{Z}$ & nd \\
\hline & Exomalopsis auropilosa Spinola & 18 & 0,60 & $\mathrm{Z}$ & nd \\
\hline & Melipona quadrifasciata Lepeletier & 41 & 1,36 & $\mathrm{Y}$ & nd \\
\hline & Paratrigona Schwarz sp. & 36 & 1,19 & $\mathrm{Y}$ & nd \\
\hline & Schwarziana quadripunctata (Lepeletier) & 743 & 24,61 & $\mathrm{~W}$ & $\mathrm{~d}$ \\
\hline & Tetragonisca angustula (Latreille) & 350 & 11,59 & $\mathrm{~W}$ & $\mathrm{~d}$ \\
\hline & Trigona spinipes (Fabricius) & 942 & 31,20 & $\mathrm{~W}$ & $\mathrm{~d}$ \\
\hline & Xylocopa frontalis (Olivier) & 1 & 0,03 & $\mathrm{Z}$ & nd \\
\hline \multirow[t]{9}{*}{ Halictidae } & Augochloropsis Cockerell & & & & \\
\hline & sp.1 & 1 & 0,03 & $\mathrm{Z}$ & nd \\
\hline & sp.2 & 1 & 0,03 & $\mathrm{Z}$ & nd \\
\hline & sp.3 & 1 & 0,03 & $\mathrm{Z}$ & nd \\
\hline & sp.4 & 2 & 0,07 & $\mathrm{Z}$ & nd \\
\hline & sp.5 & 2 & 0,07 & $\mathrm{Z}$ & nd \\
\hline & TOTAL & 3019 & & & \\
\hline & $\mathrm{H}^{\prime}$ & 1,58 & & & \\
\hline & $\mathrm{J}$ & 0,51 & & & \\
\hline
\end{tabular}


Tabela 2 - Análise de sobreposição do horário de forrageamento entre as espécies predominantes da cultura de urucum (Bixa orellana L.), estimado a partir da porcentagem de sobreposição.

\begin{tabular}{lccc}
\hline & S. quadripunctata & T. angustula & Trigona spinipes \\
\hline Apis mellifera & 81,4 & 83 & 89,5 \\
Schwarziana quadripunctata & & 93,7 & 78,8 \\
Tetragonisca angustula & & & 79,9 \\
\hline
\end{tabular}

dos recursos entre as espécies ao longo do dia (Figura 1). Quatro espécies foram consideradas acessórias (Bombus morio, Centris cfr. collaris, Melipona quadrifasciata e Paratrigona sp.) e as demais 14 espécies consideradas acidentais, com um total de indivíduos coletados por espécie variando de 1 a 23. ALMEIDA \& PINHEIRO (1992), em Viçosa, MG constataram que os polinizadores efetivos do urucum são as abelhas de grande porte Xylocopa frontalis, Centris sp., Eulaema nigrita e E.cingulata. A flor do urucum apresenta anteras com deiscência poricida, o que limita as espécies polinizadoras àquelas que são capazes de vibrar as anteras para obter grãos de pólen (LARSON \& BARRET, 1999; BEZERRA \& MACHADO, 2003; OLIVEIRA-REBOUÇAS \&
GIMENES, 2004; SILVA et al., 2004). A presença de espécies como Bombus morio, Centris aenea, $C$. c fr. collaris, C. obsoleta, Eulaema nigrita, Oxeae flavescens e Xylocopa frontalis, que são de grande porte, poderiam ser consideradas no manejo da cultura do urucum. Entretanto, com exceção de B. morio e $\boldsymbol{C}$. cfr collaris, que foram consideradas acessórias, as demais são acidentais nas coletas, o que não favoreceria o papel de agente polinizador da cultura neste estudo.

Os dados revelaram uma maior atividade de abelhas nas flores dos horários 8-10h e 10-12h, quando aproximadamente $71 \%$ dos indivíduos foram coletados nessa faixa de horário. A atividade das abelhas nas flores pode estar relacionada a algumas questões como ritmo de atividade dos indivíduos,

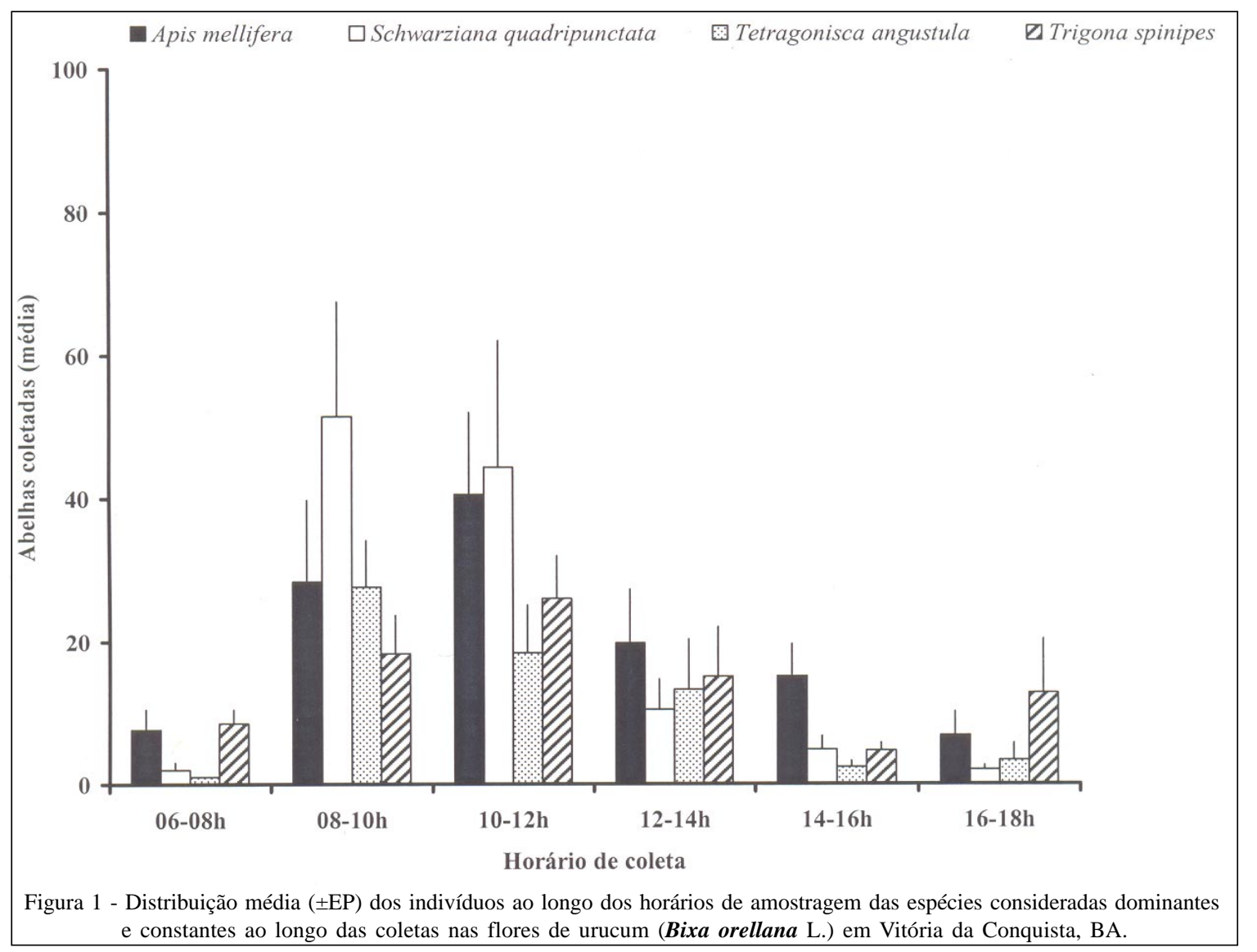

Ciência Rural, v.38, n.2, mar-abr, 2008. 
disponibilidade dos recursos nas flores ao longo do dia e também fatores físicos como temperatura e insolação. ALMEIDA \& PINHEIRO (1992) afirmam que o clímax de floração do urucum ocorre entre 10h e 16h, coincidindo com o período de temperatura mais elevada, em Viçosa, MG. Dessa maneira, a atividade de forrageamento nas flores estaria relacionada com o maior oferecimento de recursos explorados pelas abelhas, fato esse que ainda deve ser confirmado para as condições em Vitória da Conquista.

O presente trabalho registrou uma maior atividade das abelhas nas flores do urucum pela manhã (das $8 \mathrm{~h}$ às $12 \mathrm{~h}$ ), para as condições de Vitória da Conquista, devendo-se considerar esses horários para eventuais tratamentos com agrotóxicos na cultura.

\section{REFERÊNCIAS}

ALMEIDA, E.C.; PINHEIRO, A.L. Biologia floral e mecanismo de reprodução em urucuzeiro (Bixa orellana L.) I. tipo "fruto verde piloso”. In: REUNIÃO TÉCNICO CIENTÍFICA SOBRE O MELHORAMENTO GENÉTICO DO URUCUZEIRO, 1991, Belém, PA. Anais... Belém: EMBRAPA - CPATU, 1992. v.1, p.72-81.

BEZERRA, E.L. de S.; MACHADO, I.C. Biologia floral e sistema de polinização de Solanum stramonifolium Jacq. (Solanaceae) em remanescentes de mata atlântica, Pernambuco. Acta Botânica Brasileira, São Paulo, v.2, n.17, p.247-257, 2003.

GÓMEZ, M.C.E. Estudio de la biologia floral del achiote Bixa orellana L. 1980. 80f. Monografia (Graduação em
Agronomia) - Curso de Agronomia, Faculdade de Ciencias Agropecuarias de Palmira/ Universidade Nacional de Colombia, Palmira.

KREBS, C.J. Niche overlap and diet analysis. In:

Ecological methodology. New York: Harper Collins, 1989. Cap.11, p.371-407.

LARSON, B.M.H.; BARRETT, S.C.H. The ecology of pollen limitation in buzz-polinated Rhexia virginica (Melastomataceae). Journal of Ecology, London, v.87, p.371381, 1999.

JOLY, A.B. Botânica. Introdução à taxonomia vegetal. São Paulo: Companhia Editora Nacional, 1991. 777p.

OLIVEIRA-REBOUÇAS, P.; GIMENES, M. Abelhas (Apoidea) visitantes de flores de Comolia ovalifolia DC Triana (Melastomataceae) em uma área de restinga na Bahia. Neotropical Entomology, Londrina, v.33, n.3, p.315-320, 2004.

SILVA da, A.C. et al. Pollen morphology and study of the visitors (Hymenoptera, Apdae) of Solanum stramoniifolium Jacq. (Solanaceae) in central amazon. Acta Botânica Brasileira, São Paulo, v.3, n.18, p.653-657, 2004.

SILVEIRA NETO. S. et al. Manual de ecologia dos insetos. Piracicaba: Ceres, 1976. 419p.

VILARES, A.S. et al. Estudo da biologia floral de urucueiro (Bixa orellana L.). Revista Brasileira de Corantes Naturais, Viçosa, v.1, n.1, p.101-105, 1992. 